\title{
The evolving structure of the Cd isotopes
}

\author{
P.E. Garrett ${ }^{1, a}$ \\ ${ }^{1}$ Department of Physics, University of Guelph, Guelph, ON, N1G2W1 Canada
}

\begin{abstract}
The even-even Cd isotopes have long been cited as one of the prime examples of vibrational behaviour, identified soon after the Bohr model was developed in the early 1950's. By the late 1970's, the presence of intruder states and shape coexistence were identified, but the underlying vibrational nature remained intact. More recently, the robustness of the multiphonon states was questioned, prompting detailed spectroscopic investigations at a number of facilities, including the use of the $\left(n, n^{\prime} \gamma\right)$ reaction and $\beta$ decay studies with modern $\gamma$-ray spectrometers. Combining results from these studies, a re-examination of the structure of the mid-shell $\mathrm{Cd}$ isotopes suggests that they may represent deformed $\gamma$-soft rotors rather than spherical vibrators.
\end{abstract}

\section{The early years: Cd as spherical vibrators}

The collective model of Bohr and Mottelson [1,2], developed in the early 1950's, is one of the foundational models of nuclear structure. Its three main paradigms, deformed-rotational, spherical vibrational, and $\gamma$-soft, remain to this day benchmarks against which structure of nuclei are compared. Soon after the development of the vibrational limit of the Bohr Hamiltonian, in 1955 Scharff-Goldhaber and Weneser [3] used the ratio of the energy of the second excited state to the first excited state, $E_{2} / E_{1}$, to identify candidates for spherical vibrators. In this early work, the $\mathrm{Cd}$ nuclei ${ }^{110} \mathrm{Cd}$ and ${ }^{114} \mathrm{Cd}$ were cited as candidates, having $E_{2} / E_{1} \approx 2$. Soon thereafter, in 1956 [4], spectroscopy following the $(n, \gamma)$ capture reaction identified an additional $2^{+}$level in the vicinity of the two-phonon triplet in ${ }^{114} \mathrm{Cd}$. This was seen as a problem for the vibrational model by Cohen and Price [5], who, in 1960, using the $(d, p)$ reaction, not only confirmed the additional $2^{+}$level in ${ }^{114} \mathrm{Cd}$, but also found an extra $0^{+}$state. Further, they identified two $0^{+}$and two $2^{+}$states in ${ }^{112} \mathrm{Cd}$ at twice the energy of the $2_{1}^{+}$state. The validity of the phonon model was supported, however, by a series of Coulomb excitation experiments by McGowan and co-workers [6], results of which are shown in Fig. 1, that determined in ${ }^{112,114,116} \mathrm{Cd}$ the ratio $B\left(E 2 ; 4_{1}^{+} \rightarrow 2_{1}^{+}\right) / B\left(E 2 ; 2_{1}^{+} \rightarrow 0_{1}^{+}\right)$was approximately equal to 2 (within the experimental uncertainty). Only in ${ }^{110} \mathrm{Cd}$ did the ratio of $1.42 \pm 0.19$ deviate significantly from 2.0. Furthermore, in ${ }^{114,116} \mathrm{Cd}$, the ratio $B\left(E 2 ; 0^{\prime+} \rightarrow 2_{1}^{+}\right) / B\left(E 2 ; 2_{1}^{+} \rightarrow 0_{1}^{+}\right)$was measured to be near unity, again providing support to the phonon model.

\section{Elucidation of the intruder states}

The nature of the extra states in the two-phonon region was problematic and speculated upon until, in 1977, Meyer and Peker [7] used the $\beta$-decay of the high-spin ${ }^{110}$ In ground state to populate high-spin

\footnotetext{
ae-mail: pgarrett@physics.uoguelph.ca
} 

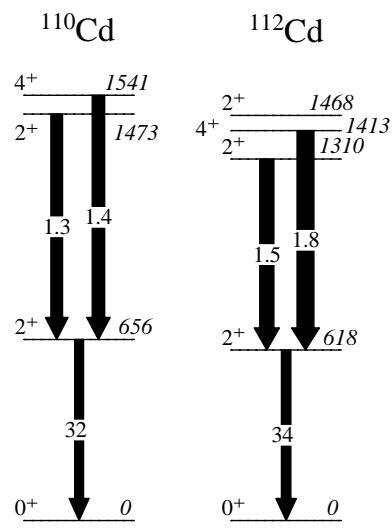

${ }^{114} \mathrm{Cd}$

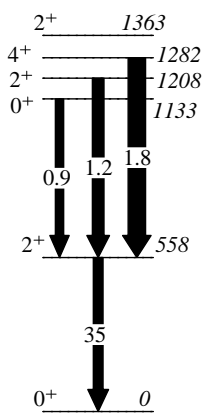

${ }^{116} \mathrm{Cd}$

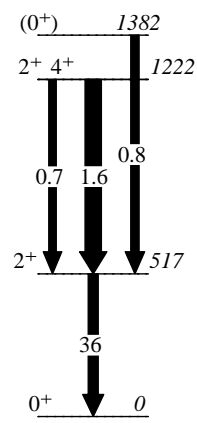

Figure 1. Results of the Coulomb excitation experiments by McGowan et al. [6]. The $B\left(E 2 ; 2_{1}^{+} \rightarrow 0_{1}^{+}\right)$values listed are in Weisskopf units, whereas those for the excited states are the ratios with respect to the $2_{1}^{+} \rightarrow 0_{1}^{+}$values. In ${ }^{116} \mathrm{Cd}$, the $2^{+}$and $4^{+}$levels are degenerate; the $B(E 2)$ values listed on the transitions are for the $2_{2}^{+}$level (left) and $4^{+}$ level (right). Uncertainties on the $B(E 2)$ ratio were in the range of $15-20 \%$. These data helped cement the assignment of the $\mathrm{Cd}$ isotopes as spherical vibrators.

states in ${ }^{110} \mathrm{Cd}$, observing a rotational-like band built on the $0_{2}^{+}$state. Citing evidence from the $\left({ }^{3} \mathrm{He}, n\right)$ two-proton transfer reactions performed on a series of Pd isotopes by Fielding et al. [8], in which the $0_{2}^{+}$levels in ${ }^{110,112} \mathrm{Cd}$ were nearly as strongly populated as the ground state, Meyer and Peker [7] speculated that the rotational-like states were built on $2 p-4 h$ proton intruder excitations, with the configuration $g_{9 / 2}^{-4} d_{5 / 2}^{2}$. Following this work, the nature of the excited $0_{2}^{+}$and $0_{3}^{+}$states in ${ }^{112,114} \mathrm{Cd}$ were investigated by Julin et al. [9], who concluded that the mixing between the $0_{2}^{+}$and $0_{3}^{+}$states must be weak, or the deformations small, based on the smallness of the $\rho^{2}\left(0_{3}^{+} \rightarrow 0_{2}^{+}\right)$value. Shortly thereafter, Heyde and coworkers [10] developed a model for ${ }^{112,114} \mathrm{Cd}$, using both the particle-core coupling model and the interacting boson model (IBM), that could reproduce the data as known at the time (1982). In that work [10], in order to reproduce the decays of the $0_{2}^{+}$and $0_{3}^{+}$levels, the mixing of the intruder and multi-phonon states was taken to be strong, with the $0_{2 p h}^{+}$and $0_{I}^{+}$wave functions being completely mixed [10] in the $0_{2}^{+}$and $0_{3}^{+}$states. Schematic calculations [11] based on this idea for the $\mathrm{Cd}$ isotopic chain reproduced the general trend of the decays and excitation energies of the two-phonon and intruder states, and predicted that in the heavier Cd isotopes the multiphonon states should have a rather pure character. In a systematic study of the $(p, t)$ ground state cross sections on the stable Cd isotopes, Bauer et al. [12] could not deduce any evidence for strong mixing between the $0^{+}$ ground states and $0^{+}$intruder states. In contrast, Fortune [13] and O'Donnell et al. [14] examined the excited $0^{+}$state to ground state $(p, t)$ cross section ratio, and using IBM expressions for the transfer amplitudes, deduced evidence for a large mixing matrix element between the ground state and $0_{I}^{+}$ intruder state, and a much weaker matrix element between the two-phonon $0_{2 p h}^{+}$and $0_{I}^{+}$states.

\section{Questioning Cd as a vibrational paradigm}

A study of the decay of ${ }^{118} \mathrm{Ag}$ suggested that ${ }^{118} \mathrm{Cd}$ was an example of a near-harmonic vibrational nucleus [15]; the requisite grouping of tightly spaced levels was observed, and the decays also strongly favoured $\Delta N=1$ transitions. Based on the systematics, the $0_{2}^{+}$level was assigned as the two-phonon level with the $0_{3}^{+}$state as the intruder band head. Lifetime measurements [16], however, did not find sufficient collectivity in the decay of the $0_{2}^{+}$level, raising some doubts about the assignments. Further questions were raised in the most detailed study of electromagnetic matrix elements in the Cd isotopes using Coulomb excitation of ${ }^{114} \mathrm{Cd}$ by Fahlander et al. [17]. In that work, the strong-mixing scenario between the excited states was deemed unlikely. The best agreement with the data assumed a highly anharmonic multiphonon structure requiring the shifting of some of the three-phonon states into the region of the two phonon states. Detailed spectroscopy of the Cd isotopes by the Fribourg [18-20] and Jyväskylä [21] groups reinforced the existence of the intruder bands, but came to different conclusions 
regarding the strength of the mixing. The ambiguity of the degree of mixing was also the conclusion reached by Casten et al. [22] in a study of the $B(E 2)$ values deduced from level lifetimes in ${ }^{114} \mathrm{Cd}$.

Beginning in the mid-1990's, a systematic study of the stable even-even Cd isotopes using the powerful $\left(n, n^{\prime} \gamma\right)$ reaction was undertaken. This reaction provides comprehensive spectroscopy and the ability to deduce level lifetimes in the $1 \mathrm{fs}-1 \mathrm{ps}$ range, yielding $B(E 2)$ values for low-spin, highly non-yrast states [23-28]. In the early studies of ${ }^{110,112} \mathrm{Cd}$ [23-25] with the $\left(n, n^{\prime} \gamma\right)$ reaction, the $B(E 2)$ values for the observed $\gamma$ rays were in line with expectations or not deemed significant. However, later studies [26-28] started to reveal more serious discrepancies, particularly highlighted in ${ }^{116} \mathrm{Cd}$ [26] where the strong-mixing calculations failed to explain the decays of the $0_{2}^{+}$and $0_{3}^{+}$levels which has the higher energy $0_{3}^{+}$intruder band head still retaining the enhanced $B\left(E 2 ; 0^{+} \rightarrow 2_{1}^{+}\right)$strength, and the lower energy $0_{2}^{+}$two-phonon state possessing a suppressed $B(E 2)$ value. Further questions were raised when it was noted that the same discrepancies occurred systematically across the Cd isotopes [29], suggesting a breakdown of vibrational motion occurring at the three-phonon level [29].

An important issue in assessing collectivity at high excitation energies is the ability to observe weak, low-energy transitions that must compete with high-energy non-collective transitions. Because of the $E_{\gamma}^{5}$ factor in the transition rate, even highly-collective low-energy $\gamma$-ray transitions can have a vanishingly small branching ratio. Fortunately, $\beta$-decay, observed with modern spectrometers, provides a solution since it (typically) has much lower background in the spectra at low $\gamma$-ray energies compared to in-beam reactions. Programmes to study the $\mathrm{Cd}$ isotopes via $\beta$ decay have been been carried out at both the Jyväskylä [30, 31] and HRIBF facilities [32, 33], focusing on the neutron-rich species, and at TRIUMF [34, 35]. These studies have provided a wealth of new data and resolved a number of issues with the level schemes. Based in part on this new data, and considering all spectroscopic information including results from transfer reactions, it was argued that the mid-shell Cd isotopes were not spherical vibrators, and the levels re-organized into rotational-like bands [36].

The most recent study of ${ }^{110} \mathrm{Cd}$, combining a re-analysis of the data from the $\left(n, n^{\prime} \gamma\right)$ reaction and the study of ${ }^{110} \operatorname{In} \beta$-decay [35] was able to observe or provide stringent limits on $\gamma$-ray branchings between the intruder states and purported multiphonon excitations. Except for the decay of the intruder $0^{+}$band head, and the $6^{+}$member of the ground state band, all other possible transitions connecting the intruder and non-intruder states were found to be weak, thereby refuting the strong-mixing scenario. With this conclusion, the near-vanishing $B\left(E 2 ; 0_{3}^{+} \rightarrow 2_{1}^{+}\right)$value and enhanced $B\left(E 2 ; 0_{3}^{+} \rightarrow 2_{2}^{+}\right)$ value, together with the staggering in the " $K^{\pi}=2^{+}$" quasi- $\gamma$ band, were recognized as strong indicators of $\gamma$-soft behaviour. The re-organization of the low-lying levels in ${ }^{110} \mathrm{Cd}$ into rotational-like bands in shown in Fig. 2. Further, the similarity of the level structures in ${ }^{112,114,116} \mathrm{Cd}$ is suggestive that they also behave as $\gamma$-soft rotors; a conclusion backed up by recent theoretical calculations [37].

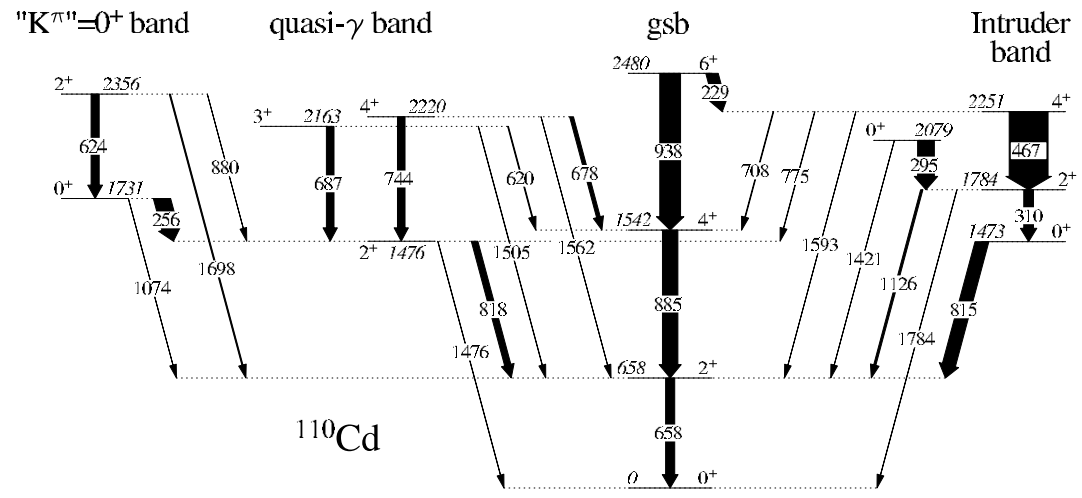

Figure 2. The re-organization of levels in ${ }^{110} \mathrm{Cd}$ into rotational bands. The enhanced decay of the $0_{3}^{+}$level to the $2_{2}^{+}$state, and the odd-even staggering observed in the $\gamma$ band, are suggestive of a $\gamma$-soft rotor. 
EPJ Web of Conferences

\section{Conclusions}

The Cd isotopes, being assigned as spherical vibrators in the 1950 's, serving as paradigms of vibrational motion for decades, are nearly 60 years later suggested to be $\gamma$-soft rotors instead. This provides an excellent example of the level of detail that is sometimes necessary to elucidate nuclear structure.

\section{References}

[1] A. Bohr, Mat. Fys. Medd. Dan. Vid. Selsk 26, no. 14 (1952)

[2] A. Bohr and B.R. Mottelson Nuclear Structure Vol. 1 (Benjamin, Reading, Mass., 1975)

[3] G. Scharff-Goldhaber and J. Weneser, Phys. Rev. 98, 212 (1955)

[4] H.T. Motz, Phys. Rev. 104, 1353 (1956)

[5] B.L. Cohen and R.E. Price, Phys. Rev. 118, 1582 (1960)

[6] F.K. McGowan, R.L. Robinson, P.H. Stelson, and J.L.C. Ford, Nucl. Phys. 66, 97 (1965)

[7] R. Meyer and L. Peker, Z. Phys. A283, 379 (1977)

[8] H.W. Fielding et al., Nucl. Phys. A281, 389 (1977)

[9] R. Julin et al., Z. Phys. A296, 315 (1980)

[10] K. Heyde et al., Phys. Rev C25, 3160 (1982)

[11] A. Aprahamian et al., Phys. Lett. B140, 22 (1984)

[12] R.W. Bauer et al., Phys. Rev. C 34, 1110 (1986)

[13] H.T. Fortune, Phys. Rev. C 35, 2318 (1987)

[14] J.M. O’Donnell, A. Kotwal, and H.T. Fortune, Phys. Rev. C 38, 2047 (1988)

[15] A. Aprahamian et al., Phys. Rev. Lett. 59, 535 (1987)

[16] H. Mach et al., Phys. Rev. Lett. 63, 143 (1989)

[17] C. Fahlander et al., Nucl. Phys. A485, 327 (1988)

[18] J. Kern et al., Nucl. Phys. A512, 1 (1990)

[19] M. Délèze et al., Nucl. Phys. A551, 269 (1993)

[20] M. Délèze et al., Nucl. Phys. A554, 1 (1993)

[21] J. Kumpulainen et al., Phys. Rev. C 45, 640 (1992)

[22] R.F. Casten et al., Phys. Lett. B297, 19 (1992)

[23] H. Lehmann et al., Phys. Lett. B387, 259 (1996)

[24] F. Corminboeuf et al., Phys. Rev. Lett. 84, 4060 (2000)

[25] F. Corminboeuf et al., Phys. Rev. C 63, 014305 (2000)

[26] M. Kadi et al., Phys. Rev. C 68, 031306(R) (2003)

[27] P.E. Garrett et al., Phys. Rev. C 75, 054310 (2007)

[28] D. Bandyopadhyay et al., Phys. Rev. C 76, 054308 (2007)

[29] P.E. Garrett, K.L. Green, and J.L. Wood, Phys. Rev. C 78, 044307 (2008)

[30] Y. Wang et al., Phys. Rev. C 67, 064303 (2003)

[31] D.L. Smith et al., Phys. Rev. C 77, 014309 (2008)

[32] J. Batchelder et al., Phys. Rev. C 80, 054318 (2009)

[33] J. Batchelder et al. Phys. Rev. C 86, 064311 (2012)

[34] K.L. Green et al., Phys. Rev. C 80, 032502 (2009)

[35] P.E. Garrett et al., Phys. Rev. C 86, 044304 (2012)

[36] P.E. Garrett and J.L. Wood, J. Phys. G 37, 064028 (2010); corrigendum ibid., 069701.

[37] L. Próchniak, P. Quentin, M. Imadalou Int. J. Mod. Phys. E21, 1250036 (2012) 